\title{
A comparison between manual and artificial intelligence-based automatic positioning in CT imaging for COVID-19 patients
}

\section{Yadong Gang}

Zhongnan Hospital of Wuhan University

\section{Xiongfeng Chen}

Puren Hospital affiliated to Wuhan University of Science and Technology

Huan Li

Zhongnan Hospital of Wuhan University

\section{Hanlun Wang}

Zhongnan Hospital of Wuhan University

Jianying Li

Computed Tomography Research Center

\section{Ying Guo}

Computed Tomography Research Center

Junjie Zeng

Zhongnan Hospital of Wuhan University

Qiang Hu

Zhongnan Hospital of Wuhan University

Jinxiang Hu

Zhongnan Hospital of Wuhan University

Haibo Xu ( $\nabla$ xuhaibo1120@hotmail.com)

Zhongnan Hospital of Wuhan University

\section{Research Article}

Keywords: Coronavirus, Artificial intelligence, Tomography, Radiation dosage

Posted Date: October 29th, 2020

DOl: https://doi.org/10.21203/rs.3.rs-57162/v2

License: (1) (1) This work is licensed under a Creative Commons Attribution 4.0 International License.

Read Full License 
Version of Record: A version of this preprint was published at European Radiology on March 19th, 2021. See the published version at https://doi.org/10.1007/s00330-020-07629-4. 


\section{Abstract}

Objective: To analyze and compare the imaging workflow, radiation dose and image quality for COVID-19 patients examined using either the conventional manual positioning (MP) method or an Al-based automatic positioning (AP) method.

Materials and Methods: 127 adult COVID-19 patients underwent chest CT scans on a CT scanner using the same scan protocol except with the manual positioning (MP group) for the initial scan and an Albased automatic positioning method (AP group) for the follow-up scan. Radiation dose, patient positioning time and off-center distance, of the two groups were recorded and compared. Image noise and signal-to-noise ratio (SNR) were assessed by three experienced radiologists and were compared between the two groups.

Results: The AP operation was successful for all patients in the AP group and reduced the total positioning time by $28 \%$ compared with the MP group. Compared with the MP group, the AP group had significantly less patient off-center distance (AP:1.56cm \pm 0.83 vs. MP: $4.05 \mathrm{~cm} \pm 2.40, p<0.001$ ) and higher proportion of positioning accuracy (AP: $99 \%$ vs. MP: $92 \%$ ), resulted in 16\% radiation dose reduction (AP: $6.1 \mathrm{mSv} \pm 1.3 \mathrm{vs}$. MP: $7.3 \mathrm{mSv} \pm 1.2, p<0.001)$ and $9 \%$ image noise reduction in erector spinae and lower noise and higher SNR for lesions in the pulmonary peripheral areas.

Conclusion: The Al-based automatic positioning and centering in CT imaging is a promising new technique for reducing radiation dose, optimizing imaging workflow and image quality in imaging the chest. This technique has important added clinical value in imaging COVID-19 patients to reduce the cross-infection risks.

\section{Key Points}

1. The Al-based automatic positioning (AP) operation was successful for all patients in our study.

2. AP method reduced the total positioning time by $28 \%$ compared with the manual positioning (MP).

3. AP method had less patient off-center distance and higher proportion of positioning accuracy than MP method, resulted in 16\% radiation dose reduction and $9 \%$ image noise reduction in erector spinae.

\section{Summary Statement}

Al-based automatic positioning results in touch-less patient handling with higher positioning efficiency and more patient centering accuracy, less radiation dose, and better image quality in chest CT imaging, which adds clinical value for diagnosing COVID-19 patients to reduce the cross-infection risks.

\section{Abbreviations}

Artificial intelligence $=\mathrm{Al}$ 
2019 coronavirus disease $=$ COVID-19

Manual positioning $=$ MP

Al-based automatic positioning $=$ AP

Signal-to-noise ratio $=$ SNR

\section{Introduction}

Accurate patient positioning and centering in computed tomography (CT) remains an important issue of concern for reducing dose and image noise[1-3]. One study reported that patients were mis-centered by 6 $\mathrm{cm}$, resulting in up to $41 \%$ surface dose and $22 \%$ image noise increase[4]. To achieve high diagnostic image quality at reduced radiation dose, technologists make an extra effort to accurately select the anatomic scan range and carefully center the patients during CT scans. However, manual positioning and centering with accuracy is a time-consuming process and technologist-dependent and often inconsistent and non-optimal. For patients with infectious diseases, the interaction between technologists and patients also carries the potential cross-infection risk.

Recent advances in artificial intelligence (Al) technologies have demonstrated remarkable progress in recognizing and interpreting complex patterns in imaging data. The combination of Al and CT imaging can provide faster, more accurate and efficient imaging-based diagnosis[5]. By virtue of 3D visual sensors, Al can identify the pose and shape of patients and realize an automated contactless image acquisition workflow. Yang Wang et al. (2020) reported that U-HAPPY (United imaging Human Automatic Planbox for PulmonarY) CT has a function with automatic positioning and scanning, which helps to reduce the radiation dose[6]. Booij et al. [7] and Saltybaeva et al. [8] also reported the patient centering accuracy in CT using 3D cameras that relies on deep neural network for image contouring. Recently, GE Healthcare introduced a Revolution Maxima CT, which relies on deep learning algorithms and real-time depth sensing technology to center patients, locate desired anatomies and perform scan automatically. This CT scanner was successfully used for diagnosing COVID-19 patients in our hospital during the pandemic. However, applying Al to CT scanning technique is still at the exploratory stage. The purpose of this study was to analyze and compare the imaging workflow, patient positioning and centering accuracy, radiation dose and image quality of COVID-19 patients who underwent several follow-up CT scans using the same CT protocol on a same CT machine but with either the conventional manual positioning (MP) mode or an Al-based automatic positioning (AP) mode. We hope our findings may provide useful information on the characteristic of intelligent $\mathrm{CT}$ tools and help radiologists to achieve better images at lower radiation dose more efficiently, while to reduce the potential risks of medical workers exposing to patients with infectious diseases during CT examination.

\section{Materials And Methods}


The research was approved by Medical Ethical Committee (Approved Number. 2020037). Our institutional review board waived written informed consents for this study, and got consent from patients.

\section{Patients and data source}

All the patients in our study had been diagnosed of COVID-19 according to the guideline of 2019-nCoV (Fifth Trial Edition) issued by the National Health Commission of China [9]. A total of 127 patients (68 men and 59 women; mean age, 57.7 years; age range, 20-83 years) with confirmed SARS-CoV-2 were identified who had undergone at least two chest CT studies at Wuhan Leishenshan Hospital between Feb 12, 2020 and Apr 10, 2020 (see more details in Table 1). These patients underwent the first chest CT using the conventional manual positioning and centering method, and an Al-based automatic positioning and centering method in the follow-up CT examination. The patients in our study were limited to the ones without the need for life-supporting tubes and other equipment and could follow verbal command. The interval time between the two scans was 5-8 day. Based on the different positioning methods, patients were categorized into the conventional manual positioning (MP) group and Al-based automatic positioning (AP) group, and all CT images and clinical data between the two groups were compared.

\section{CT image acquisition and reconstruction}

The imaging workflows for the MP and AP groups are shown in Figure 1. A and B. The chest CT scanning was performed on a Revolution Maxima CT equipped with an Al-based automatic patient centering and anatomic positioning software (GE Healthcare, Waukesha USA) from the apex pulmonis to diaphragm. Both groups used the same scan protocol with the following parameters: tube voltage, 120 kVp; gantry rotation time, 0.4 second; pitch, 1.375:1; scan field-of-view (SFOV), 50cm; slice thickness, $5 \mathrm{~mm}$; tube current (mA), automated tube current modulation (ATCM) to obtain a noise index of 11.57; All axial images were reconstructed using a standard reconstruction algorithm with the standard kernel; reconstruction display field-of-view (DFOV), 35-50 cm; reconstruction thickness, $1.25 \mathrm{~mm}$.

\section{Al-based automatic patient positioning and centering}

The Al-based automatic positioning uses a fixed, ceiling mounted, off the shelf, 2D/3D video camera that can determine distances to points in its field of view. It displays standard RGB video images on the CT system's existing gantry-mounted touchscreens (Figure 2 A, B). Information from the standard output of the camera is used, along with precise spatial information of the individual CT system's gantry and table installation geometry, to determine the anatomical landmark location and the start and end locations for the scout scan(s). The scan protocol structure on the scanner contains a field for the anatomical reference. The 8 supported anatomical references for the automatic positioning method are: Orbital Meatal baseline (OM), Sternoclavicular Notch (SN), Xyphoid (XY), Iliac Crest (IC), Left and Right Knee $(\mathrm{KN})$, Left and Right Ankle Joint (AJ), as shown in Figure $2 \mathrm{C}$. The automatic positioning software uses two deep learning algorithms (RGBLandmarkNet network and DepthLandmarkNet network) with different inputs that produce comparable outputs to identify all 8 of the anatomical landmarks on the patient's body. All 8 of these identified landmarks are used to determine the patient orientation (head or feet first). 
In our study, the SN and IC landmarks were used for the chest scan. The RGBLandmarkNet network uses $2 \mathrm{D}$ video images as inputs and outputs all eight of the predefined landmark locations in $\mathrm{X}$ and $\mathrm{Z}$. In parallel, the DepthLandmarkNet network uses the 3D depth data from the camera to also produce all eight of the predefined landmark locations. The 3D depth images are used to generate a "point cloud" on a mesh of points on the patient surface contour as determined from the depth information. The point cloud is then segmented to produce the body contour. The body contour is used to deterministically calculate the vertical geometric center of the patient. The center point location is then used to calculate the required table elevation for patient centering. With patient on the CT scanning table, the patient position and centering can be performed automatically with the one-touch button on the console in the control room.

\section{Assessment of image quality}

The image quality was analyzed by three radiologists (H.B.X., J.X.H., Y.D.G) at a standard pulmonary display window setting (window level, -700 and window width, 1500). the pulmonary lesions and the locations of ROI for these lesions were established by consensus. The mean CT value and standard deviation (SDev) in Hounsfield Units (HU) of the aorta, trachea and erector spinae in the upper and middle thorax areas were measured by placing a $50 \mathrm{~mm}^{2}$ region-of-interest (ROI) on a homogeneous-appearing area of these structures, as shown in Figure $3 \mathrm{C}$. Three consecutive images were measured in each ROI area for each study, and the average value was determined. The mean and SDev of CT values within pulmonary lesions were also measured, as shown in Figure 3 A, B. The pulmonary lesions mainly included ground glass opacification, consolidation opacification and interstitial thickening. Other radiographic abnormality (hydrothorax, nodule or lump, cavitation or calcification, bronchiole or bronchiectasis and emphysema) were also noted. The pulmonary segments were defined by referring to the branching patterns of bronchi [10-12]. If a lesion was located in the outer one third of the lung, it was defined as peripheral, otherwise, it was defined as central. The signal-to-noise ratio (SNR) of the lesions was calculated based on the formula: $S N R=$ Mean CT values/SDev. The image noise was represented using the SDev value.

\section{Positioning time}

The positioning time was recorded by the CT technologist for each study. The positioning time was defined as the time from the patient lying on the CT examination bed to technologist finishing positioning and starting scanning.

\section{Off-center distance and positioning accuracy}

The patient off-center distance was measured using an axial CT image in the following steps: (『) select a transverse image containing manubrium and draw a horizontal line that passes through both armpits. ( $\mathbb{(})$ locate the center of the display field of view (DFOV) for the image by displaying the grid and selecting the center cross over point of the grid. ( $\nabla$ ) record the vertical distance from the center of DFOV to the horizontal line (Fig 3 D). For the positioning accuracy, a complete coverage should contain the apex 
pulmonis and diaphragm. thus, if the images of apex pulmonis and diaphragm were fully covered, the patient positioning was considered successful, otherwise, it was defined incomplete or inaccurate (Fig 4 $A, B)$.

\section{Radiation dose}

The volume CT dose index (CTDI ${ }_{\text {vol }}$ in $\mathrm{mGy}$ ) and dose length product (DLP in $\mathrm{mGy}-\mathrm{cm}$ ) were recorded from the dose report image by the $\mathrm{CT}$ technologist for each study. The effective dose (ED in mSv) of the patient was calculated based on the formula: $E D=D L P \times C f$, where the $C f$ represents the conversion factor for chest CT $(=0.014 \mathrm{mSv} / \mathrm{mGy}-\mathrm{cm})$.

\section{Statistical analyses}

Continuous variables were expressed as mean \pm SD and compared using paired-sample $t$ tests when the data were normally distributed; otherwise, the Wilcoxon signed-rank tests was used; Data distribution was tested with Shapiro-Wilk test. The categorical variables were expressed as number (percentage \%) and compared with McNemar's test. A two-tailed $P$ value of less than 0.05 was considered statistically significant. All statistical analyses were conducted with IBM SPSS software (version 22.0).

\section{Results}

\section{Baseline characteristics of objects}

A total of 127 COVID-19 patients with a mean age of 57.7 years (ranged 20-83 years) were included in our study. Among them, there were $68(53.5 \%)$ men and 59 (46.5\%) women. The ratio of man to woman was $1.15: 1$. Their body mass index (BMI) values were in the range of $17.4-33.1 \mathrm{~kg} / \mathrm{m}^{2}$, with an average value of $24.3 \mathrm{~kg} / \mathrm{m}^{2}$ (See Table 1 ).

\section{Positioning time, accuracy and off-center distance comparison}

The positioning time in the AP group was significantly shorter than the MP group: $29.0 \mathrm{~s} \pm 7.0$ in AP vs. $40.0 \mathrm{~s} \pm 11.0$ in MP, $p<0.001$ (Fig 1 C). The positioning accuracy and off-center distance were determined using patient chest CT images. A significantly higher positioning accuracy was recorded in the AP group (126 of $127,99.0 \%$ ) than in the MP group (117 of 127, $92.0 \%$ ) (Fig 4). The patient off-center distances obtained with the Al-based method were all significantly less than those obtained with the manual method (mean off-center distance: $1.56 \mathrm{~cm} \pm 0.83$ in the AP group vs. $4.05 \mathrm{~cm} \pm 2.40$ in the MP group, $p<0.001)$ (Table 2).

\section{Radiation dose}

The Al-based positioning group had significantly lower CTDIvol value $(13.3 \mathrm{mGy} \pm 2.4$ vs. $14.9 \mathrm{mGy} \pm 2.3$, $p<0.001)$, DLP value (437.4mGy.cm \pm 95.6 vs. $523.4 \mathrm{mGy} . \mathrm{cm} \pm 87.7, p<0.001)$ and ED value $(6.1 \mathrm{mSv} \pm 1.3$ vs. $7.3 \mathrm{mSv} \pm 1.2, p<0.001$ ) than the manual positioning group (Table 2 ). 


\section{Image noise}

The image noise was represented by the SDev measurement of the erector spinae in the upper and middle thorax areas. In both areas, the noise levels in CT images obtained with the AP method were all statistically lower than those in CT images obtained with the MP method: mean noise in the upper thorax, AP: $49.7 \mathrm{HU} \pm 7.3$ vs. MP: $54.1 \mathrm{HU} \pm 9.3$; and mean noise in the middle thorax, AP: $48.9 \mathrm{HU} \pm 8.5 \mathrm{vs}$. MP: $53.9 \mathrm{HU} \pm 9.1$, (both $p<0.001$ ) (Table 3). However, there was no significant difference for the noise values of the aorta and trachea in both groups.

\section{Noise and SNR of pulmonary lesions}

The pulmonary lesions could be found in any pulmonary segments in both groups. However, they predominantly distributed in the peripheral area of the lungs (766 of 791 lesions in the MP group, vs. 927 of 957 lesions in the AP group) (Table 4). Overall, the AP group had marginally lower image noise and higher SNR for the lesions from the pulmonary segment point of view (Table 4). But for lesions located in the peripheral area, the AP group had significantly lower noise and higher SNR than the MP group (Table 5, Fig 3).

\section{Discussion}

We analyzed and compared the imaging workflow, radiation dose and image quality for COVID-19 patients examined using either the conventional manual positioning method or an Al-based automatic patient positioning and centering method. Our results indicated that the Al-based method not only automatically positioned patients with $99 \%$ accuracy and reduced the patient positioning time, but also reduced the radiation dose to patients and overall image noise by better centering the patients and with less positioning error margin.

Achieving high image quality at reduced radiation dose is always desirable. Reducing patient examination time and quickly diagnosing disease becomes even more necessary during the COVID-19 pandemic, because quickly screening and treating the patients should be the most critical measures for containing the pandemic. Introducing artificial intelligence into CT imaging provides us a new way to achieve it. The auto-positioning function automatically detects an anatomical landmark by deep learning algorithms and allows minimizing positioning actions into a single click operation, as illustrated in figures 1 and 2. This automatic positioning operation was approved to be efficient in our research, which plays an essential role in helping the response to the COVID-19 pandemic. Our results showed that the use of Al-based positioning for chest CT scanning resulted in a shorter time to complete the patient positioning. In particular, the chest positioning time was reduced in the AP group by $28 \%$, as compared with the MP group. This automatic patient positioning operation was also approved to be accurate in our study. In our study, only one patient (1 out of 127) in the AP group required minor manual adjustment for the scan range after the Al selection. In addition, the scan range was more precise and was reduced by $6 \%$ overall based on the DLP report in the AP group which contributed to the additional $6 \%$ dose reduction for the patients in the AP group. 
According to some related researches, off-center positioned patients substantially increase image noise and dose requirement[13]. In the Al-based automatic patient positioning and centering technique, the 3D camera detects a depth information of patients and calculates the required table elevation to set the centering in the selected protocol. The auto centering function optimizes the radiation dose and image quality without of regard to operator's skill. Our research found that the patient off-center distances with the manual positioning method were more than those with the Al-based method. In our study, the patient off-center distance was substantially reduced from the $4.05 \mathrm{~cm} \pm 2.40$ in the MP group to $1.56 \mathrm{~cm} \pm 0.83$ in the AP group. The off-center position reduction in the AP group subsequently reduced the radiation dose (CTDI) requirement to achieve similar image noise by $11 \%$. Together with the tightened scan range brought about with the Al-based positioning, we achieved 16\% dose reduction in the AP group (AP: $6.1 \mathrm{mSv} \pm 1.3$ vs. MP: $7.3 \mathrm{mSv} \pm 1.2$ ).

When patients are mispositioned in the gantry, not only the radiation dose requirement is artificially increased, the image quality often underperforms as well [14]. Our results also indicated that the noise level in CT images obtained with AP mode, particularly in the erector spinae and the lesions in the peripheral lung regions, were statistically lower than those in CT images obtained with MP mode. Chung et al. [15] reported that the lung lesions in COVID-19 patients are predominantly distributed in the peripheral region of the lungs. Hence the centered patients with AP mode may have the positive impact on image quality of peripheral lesions and provide potential dose reduction opportunities.

Recently, Yang Wang et al. reported the use of an intelligent system (U-HAPPY CT)[6] to reduce radiation exposure in chest CT application. Our results showed very similar radiation exposure reduction findings. Booij et al. [7] and Saltybaeva et al. [8] also reported the patient centering accuracy in CT using 3D cameras that relies on deep neural network for image contouring. Our results also agreed with their conclusions that the Al-based technique improved patient centering accuracy, and in turn improved image quality. In addition, we also demonstrated that the Al-based patient positioning technique on our CT scanner was able to position the patients automatically with one click of the button and no human contact that not only reduced positioning time, improved workflow but also minimize the potential crosscontamination between patients and medical workers, which is even more relevant during epidemic or pandemic such as COVID-19.

Our research had some limitations. Firstly, since this scanner was purchased specifically for combating the COVID-19 pandemic, the patients were limited to COVID-19 patients due to the safety requirement, our study may suffer from confounding bias due to the relatively small number of patients. But the Al-based positioning method should not be limited to COVID-19 patients, and more evaluation with larger number of patients is needed to generalize the conclusions. In addition, amidst the fear and confusion during pandemic, the scan protocols for the newly purchased CT scanner was not fully optimized and iterative reconstruction algorithms were not used because we were not sure how the iterative reconstruction algorithms would interact with the image quality and diagnosis of COVID-19 patients. Specifically, the radiation doses used for the COVID-19 patients were on the high end of the dose spectrum and left a lot of room for improvement. However, we believe the radiation dose should not affect the conclusions of our 
study. Secondly, we only evaluated one CT scanner from one manufacturer. Additional studies are needed to investigate the generality of Al positioning on different CT scanners. Thirdly, Although the measurements in this study were performed on the same machine in the same patient, there were still differences in the status of the patient compliance during the two CT examinations.

In summary, our study indicates that the use of Al-based automatic patient positioning and centering results in less radiation dose, higher examination efficiency, higher positioning accuracy and higher image quality in CT imaging the chest. This technique has important added clinical value for diagnosing infectious patients such as COVID-19 patients to reduce the cross-infection risks between patients and medical workers.

\section{Acknowledgements}

This study was supported by the National Key Research and Development Plan of China (Grants No. 2017YFC0108803), the National Natural Science Foundation of China (Grants No. 81771819 and No. 81801667). We thank Yunxiao Han and Yinghong Ge for assistance with the collecting image data. We thank Drs. Shuai Zhang, Zhusha Wang, Chenhong Fan, Wenbo Sun, Gao Lei and other members of the Department of Radiology, Zhongnan Hospital of Wuhan University for their help with comments and advice for revising the manuscript. Guarantors of integrity of entire study, Haibo Xu, MD, PhD and Yadong Gang, PhD.

\section{References}

1. Marsh RM, Silosky MS (2017) The effects of patient positioning when interpreting CT dose metrics: a phantom study. Med Phys 44(4):1514-1524. doi: 10.1002/mp.12137

2. Ohno Y, Koyama H, Seki S, Kishida Y, Yoshikawa T (2019) Radiation dose reduction techniques for chest CT: Principles and clinical results. Eur J Radiol 111:93-103. doi: 10.1016/j.ejrad.2018.12.017

3. Saltybaeva N, Alkadhi H (2017) Vertical off-centering affects organ dose in chest CT: evidence from Monte Carlo simulations in anthropomorphic phantoms. Med Phys 44(11):5697-5704. doi:10.1002/mp.12519

4. Toth T, Ge Z, Daly MP (2007) The influence of patient centering on CT dose and image noise. Med Phys 34(7):3093-3101. doi:10.1118/1.2748113

5. Shi F, Wang J, Shi J et al. (2020) Review of artificial intelligence techniques in imaging data acquisition, segmentation and diagnosis for covid-19. IEEE Rev Biomed Eng. doi: 10.1109/RBME.2020.2987975

6. Wang Y, Lu X, Zhang Y et al. (2020) Precise pulmonary scanning and reducing medical radiation exposure by developing a clinically applicable intelligent CT system: Toward improving patient care. EBioMedicine 54:102724. doi: 10.1016/j.ebiom.2020.102724

7. Booij R, van Straten M, Wimmer A et al. (2020) Automated patient positioning in CT using a 3D camera for body contour detection: accuracy in pediatric patients. Eur Radiol. 
https://doi.org/10.1007/s00330-020-07097-w

8. Saltybaeva N, Schmidt B, Wimmer A et al. (2018) Precise and automatic patient positioning in computed tomography: avatar modeling of the patient surface using a 3-dimensional camera. Invest Radiol 53(11): 641-646. doi: 10.1097/RLI.000000000000048

9. China National Health Committee (2020) Diagnosis and treatment of pneumonitis caused by novel coronavirus (trial fifth edition). China National Health Commission, China.http://www.nhc.gov.cn/xcs/zhengcwj/202003/46c9294a7dfe4cef80dc7f5912eb1989.shtml. Accessed 4 Feb 2020

10. Foster-Carter A, Hoyle C (1945) The segments of the lungs; a commentary on their investigation and morbid radiology. Dis Chest 11(6):511-564. doi: 10.1378/chest.11.6.511

11. Sealy WC, Connally SR, Dalton ML (1993) Naming the bronchopulmonary segments and the development of pulmonary surgery. Ann Thorac Surg 55(1):184-188. doi: 10.1016/00034975(93)90507-e

12. Ugalde P, de Jesus Camargo J, Deslauriers J (2007) Lobes, fissures, and bronchopulmonary segments. Thorac Surg Clin 17(4):587-599. doi: 10.1016/j.thorsurg

13. Szczykutowicz TP, DuPlissis A, Pickhardt PJ (2017) Variation in CT number and image noise uniformity according to patient positioning in MDCT. AJR Am J Roentgenol 208(5):1064-1072. doi: 10.2214/AJR.16.17215

14. Habibzadeh M, Ay MR, AsI AK, Ghadiri H, Zaidi H (2012) Impact of miscentering on patient dose and image noise in x-ray CT imaging: phantom and clinical studies. Phys Med 28(3):191-199. doi: 10.1016/j.ejmp.2011.06.002

15. Chung M, Bernheim A, Mei X et al. (2020) CT imaging features of 2019 novel coronavirus(2019nCoV). Radiology 295(1):202-207. doi: 10.1148/radiol.2020200230

\section{Conflict Of Interest}

The authors of this manuscript declare no relationships with any companies, whose products or services may be related to the subject matter of the article. The authors declare that there are no conflicts of interest related to this article.

\section{Tables}

Table 1. Demographics and baseline characteristics of 127 COVID-19 patients included in this study. 


\begin{tabular}{|ll|}
\hline Characteristics & Patients $(\boldsymbol{n = 1 2 7})$ \\
\hline Age (years) & \\
\hline Mean \pm SD & $58 \pm 12$ \\
\hline Range (years) & $20-83$ \\
\hline Sex & $68(53.5 \%)$ \\
\hline male & $59(46.5 \%)$ \\
\hline female & $1.15: 1$ \\
\hline Ratio of male to female & \\
\hline Body mass index $\left(\mathrm{BMI}, \mathrm{kg} / \mathrm{m}^{2}\right)$ & \\
\hline Mean \pm SD & $24.3 \pm 3.2$ \\
\hline Range & $17.4-33.1$ \\
\hline
\end{tabular}

Continuous value was presented as mean \pm standard deviation (SD)

Table 2. Noise and SNC from pulmonary lesions with MP and AP on each pulmonary segment. 


\begin{tabular}{|c|c|c|c|c|c|c|c|}
\hline \multirow{2}{*}{$\begin{array}{l}\text { Location } \\
\text { (Segment) }\end{array}$} & \multirow{2}{*}{$\begin{array}{l}\text { All lesions } \\
\text { ( } n, \mathrm{MP} / \mathrm{AP})\end{array}$} & \multicolumn{2}{|l|}{ Noise } & \multicolumn{2}{|l|}{ SNC } & \multicolumn{2}{|c|}{$P$ value } \\
\hline & & MP & AP & MP & AP & Noise & SNC \\
\hline \multicolumn{8}{|l|}{ Upper lobe } \\
\hline \multicolumn{8}{|l|}{ Apicale } \\
\hline Right & $45 / 55$ & $171.0 \pm 80.5$ & $160.9 \pm 75.3$ & $4.9 \pm 3.2$ & $5.2 \pm 3.1$ & 0.522 & 0.508 \\
\hline Left & $N A^{*}$ & NA & NA & NA & NA & - & - \\
\hline \multicolumn{8}{|l|}{ Posterius } \\
\hline Right & $35 / 47$ & $157.2 \pm 75.0$ & $148.3 \pm 67.3$ & $5.2 \pm 3.5$ & $5.6 \pm 3.4$ & 0.582 & 0.589 \\
\hline Left & $57 / 59$ & $156.9 \pm 71.4$ & $132.8 \pm 62.9$ & $5.0 \pm 3.1$ & $5.6 \pm 3.5$ & 0.05 & 0.35 \\
\hline \multicolumn{8}{|l|}{ Anterius } \\
\hline Right & $46 / 55$ & $149.1 \pm 71.8$ & $129.6 \pm 62.7$ & $5.0 \pm 2.9$ & $5.6 \pm 2.9$ & 0.153 & 0.248 \\
\hline Left & $37 / 41$ & $142.3 \pm 55.7$ & $125.8 \pm 56.2$ & $5.3 \pm 2.5$ & $6.4 \pm 2.7$ & 0.199 & 0.059 \\
\hline \multicolumn{8}{|c|}{ Middle lobe } \\
\hline Mediale & $29 / 41$ & $144.7 \pm 60.8$ & $143.9 \pm 78.3$ & $5.5 \pm 3.0$ & $5.9 \pm 3.2$ & 0.962 & 0.595 \\
\hline Laterale & $43 / 53$ & $154.0 \pm 67.3$ & $135.8 \pm 60.9$ & $5.2 \pm 3.5$ & $5.8 \pm 3.3$ & 0.169 & 0.42 \\
\hline \multicolumn{8}{|l|}{ Lingulare } \\
\hline Superius & $27 / 31$ & $124.9 \pm 61.6$ & $104.2 \pm 45.6$ & $5.3 \pm 2.4$ & $6.1 \pm 2.6$ & 0.149 & 0.258 \\
\hline Inferius & $42 / 52$ & $147.8 \pm 70.7$ & $130.1 \pm 64.5$ & $5.2 \pm 2.8$ & $6.2 \pm 2.7$ & 0.208 & 0.156 \\
\hline \multicolumn{8}{|l|}{ Lower lobe } \\
\hline \multicolumn{8}{|l|}{ Superius } \\
\hline Right & $57 / 71$ & $144.1 \pm 59.9$ & $141.7 \pm 65.5$ & $5.5 \pm 3.8$ & $6.0 \pm 4.1$ & 0.834 & 0.474 \\
\hline Left & $49 / 59$ & $148.8 \pm 56.4$ & $137.0 \pm 55.1$ & $5.1 \pm 2.9$ & $5.8 \pm 3.0$ & 0.277 & 0.224 \\
\hline \multicolumn{8}{|c|}{ Basale anterius } \\
\hline Right & $42 / 49$ & $148.1 \pm 69.1$ & $130.8 \pm 73.8$ & $5.0 \pm 2.8$ & $5.2 \pm 2.7$ & 0.252 & 0.751 \\
\hline Left & $46 / 52$ & $149.7 \pm 66.5$ & $124.3 \pm 60.6$ & $5.3 \pm 3.4$ & $6.3 \pm 3.0$ & 0.051 & 0.15 \\
\hline \multicolumn{8}{|c|}{ Basale mediale } \\
\hline Right & $31 / 35$ & $155.4 \pm 64.0$ & $152.8 \pm 62.6$ & $5.0 \pm 3.0$ & $5.4 \pm 2.8$ & 0.87 & 0.664 \\
\hline Left & $N A^{\star}$ & NA & NA & NA & NA & - & - \\
\hline
\end{tabular}




\begin{tabular}{|llllllll|} 
Right & $44 / 56$ & $154.1 \pm 65.0$ & $143.6 \pm 58.3$ & $5.1 \pm 2.9$ & $5.4 \pm 3.1$ & 0.399 & 0.554 \\
\hline Left & $51 / 63$ & $164.5 \pm 64.2$ & $145.3 \pm 54.0$ & $4.5 \pm 2.7$ & $5.4 \pm 3.0$ & 0.086 & 0.129 \\
\hline Basale posterius & & & & & & & \\
\hline Right & $54 / 69$ & $158.9 \pm 62.2$ & $146.9 \pm 58.1$ & $5.1 \pm 2.7$ & $5.5 \pm 3.5$ & 0.271 & 0.504 \\
\hline Left & $56 / 69$ & $146.4 \pm 71.7$ & $131.3 \pm 71.6$ & $5.4 \pm 3.0$ & $6.5 \pm 3.6$ & 0.242 & 0.072 \\
\hline & & & & & & & \\
\hline
\end{tabular}

* NA are presented as not applicable. Data are mean \pm SD, where $n$ is the amount of the pulmonary lesions with available data. MP: Manual positioning; AP: Al-based positioning; SNR: Signal-to-noise ratio.

Table 3. Distribution of noise and SNR with MP and AP on particular lesion location at chest CT.

\begin{tabular}{|llllllll|}
\hline Lesion location & $\begin{array}{l}\text { All lesions } \\
(n, \text { MP/AP })\end{array}$ & Noise & & \multicolumn{2}{c}{ SNC } & \multicolumn{3}{c|}{$P$ value } \\
\cline { 3 - 9 } & & MP & AP & MP & AP & Noise & SNC \\
\hline Central & $25 / 30$ & $135.7 \pm 54.1$ & $132.6 \pm 59.4$ & $6.3 \pm 2.7$ & $6.6 \pm 3.2$ & 0.819 & 0.739 \\
\hline Peripheral & $766 / 927$ & $151.4 \pm 66.9$ & $137.5 \pm 64.2$ & $5.3 \pm 3.3$ & $6.1 \pm 3.6$ & $<0.001$ & $<0.001$ \\
\hline
\end{tabular}

Data are mean \pm SD, where $n$ is the amount of the pulmonary lesions with available data. MP: Manual positioning; AP: Al-based positioning; SNR: Signal-to-noise ratio.

\section{Figures}
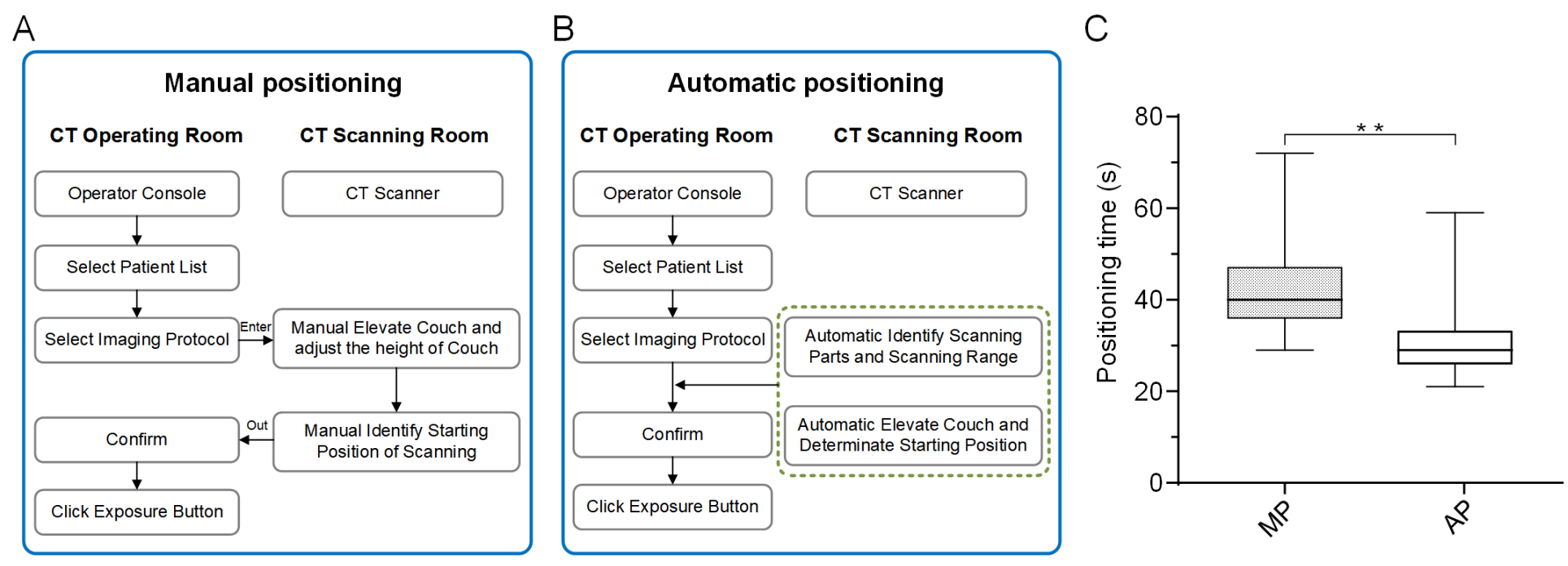

Figure 1 
Schematic diagram for the operating steps of the manual positioning and automatic positioning. (A) Flowchart for the manual positioning. (B) Flowchart for the automatic positioning. (C) Quantification of positioning time. Statistical p-value was calculated using a Wilcoxon signed-rank tests. In box plots, the central mark represents the median, and the edges of the box are the 25 th and 75 th percentiles. ${ }^{*}$ denote $p<0.01, n=127$ each.

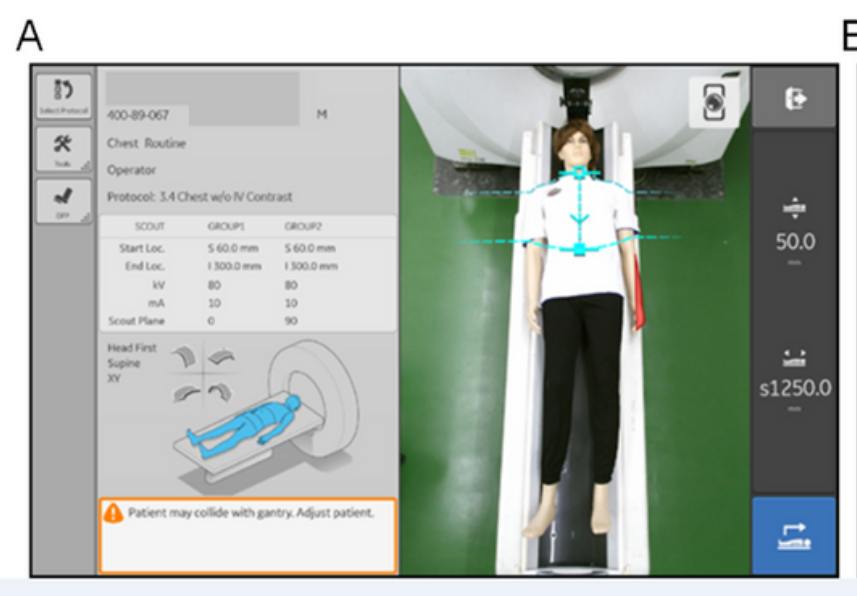

B

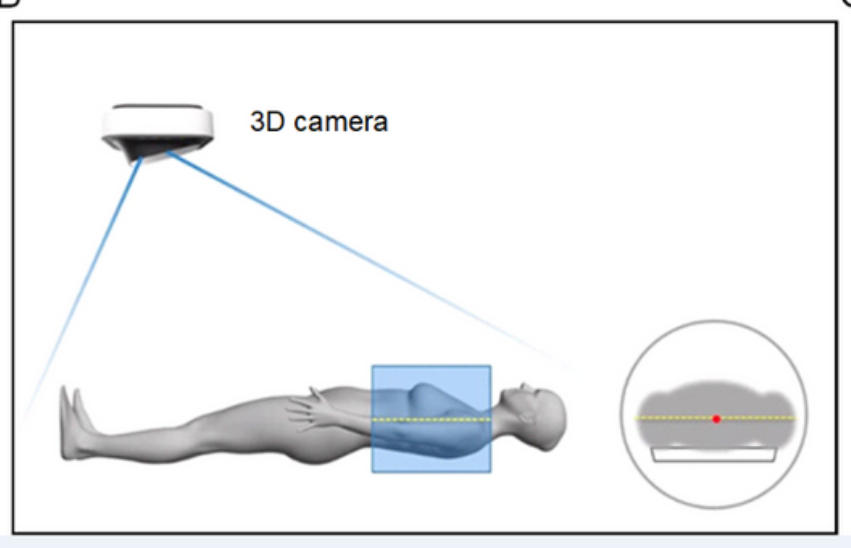

C

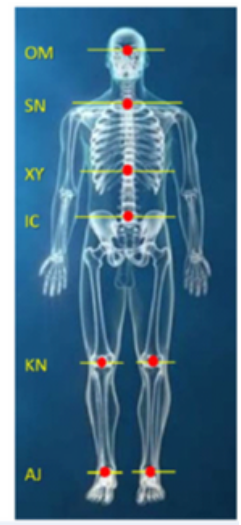

Figure 2

Schematic diagram of automatic positioning principle. (A) Al Positioning uses a fixed, ceiling mounted, off the shelf, 2D/3D video camera that can determine distances to points in its field of view. When the user selects their desired protocol, the Auto Positioning function uses the anatomical references and the scout range information to determine the landmark and the scan start and stop locations. (B) In the Albased automatic patient positioning and centering technique, the 3D camera detects a depth information of patients and calculates the required table elevation through reading the dot pattern, capturing the infrared image to set the centering in the selected protocol and achieve accurate positioning. (C) The 8 supported anatomical references / landmarks. 

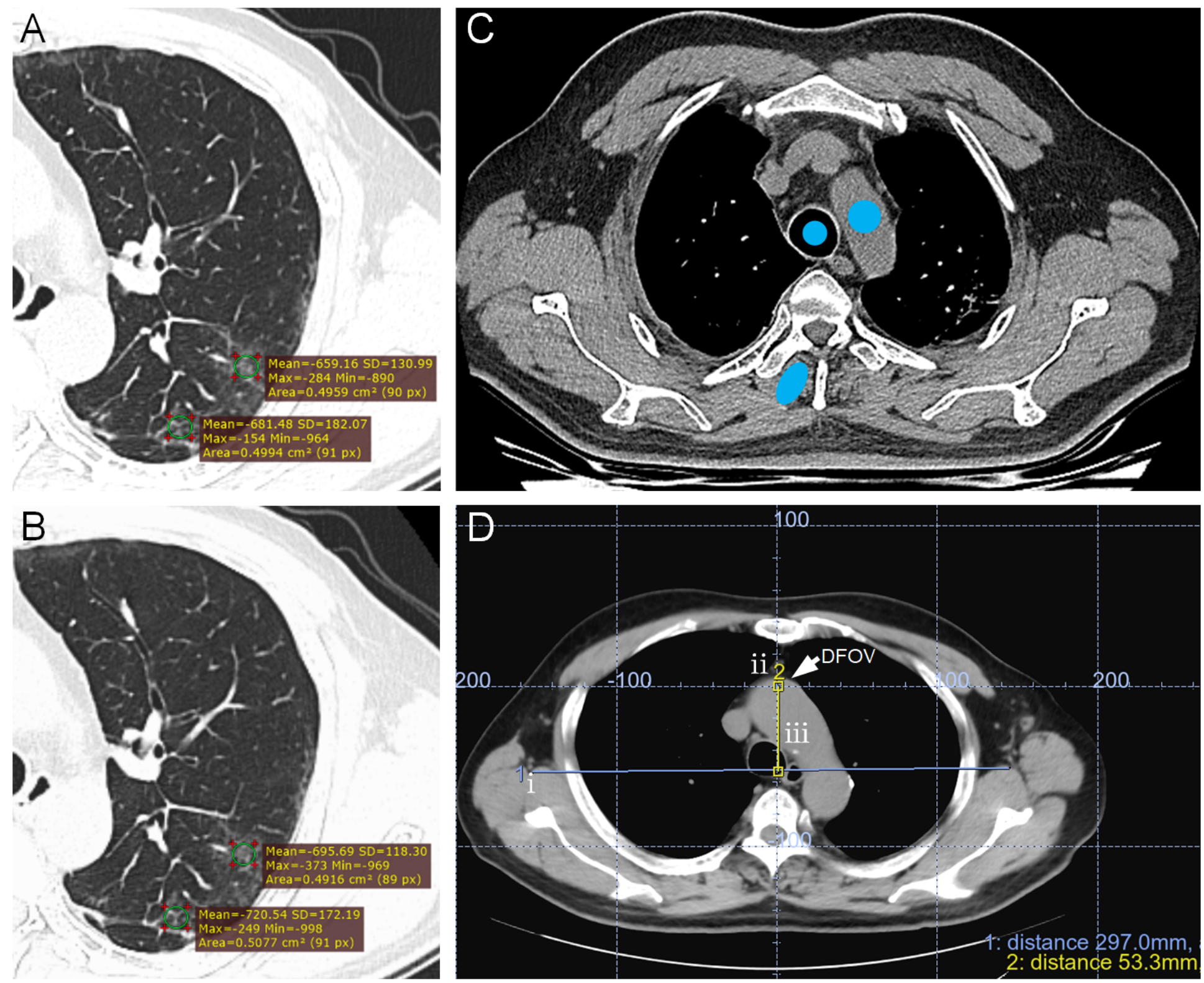

Figure 3

The measurement of CT value, noise and off-center distance on axial thin-section CT image in a COVID19 patient. lesions are shown along with ROI locations (green circles) used to acquire CT value measurements (mean \pm SD) in different lung segments. The same patient went through two CT scanning with first with MP shown in A and second with AP after six days shown in $B$, demonstrating reduced lesion noise with AP method. (C) Axial CT slice of thorax is shown along with ROI locations used to acquire CT value measurements. the mean CT values and standard deviations (SDev) was calculated by drawing a $50 \mathrm{~mm} 2$ blue circular ROI in a homogeneous-appearing area of aorta, trachea and blue oval $\mathrm{ROI}$ in erector spinae in the chest area. (D) Measurement of off-center distance: ( $)$ ) select a transverse image containing manubrium and draw a horizontal line that passes through both armpits. (囚) locate the center of the display field of view (DFOV) for the image by displaying the grid and selecting the center cross over point of the grid. ( $\mathbb{\nabla})$ record the vertical distance from the center of DFOV to the horizontal line. 

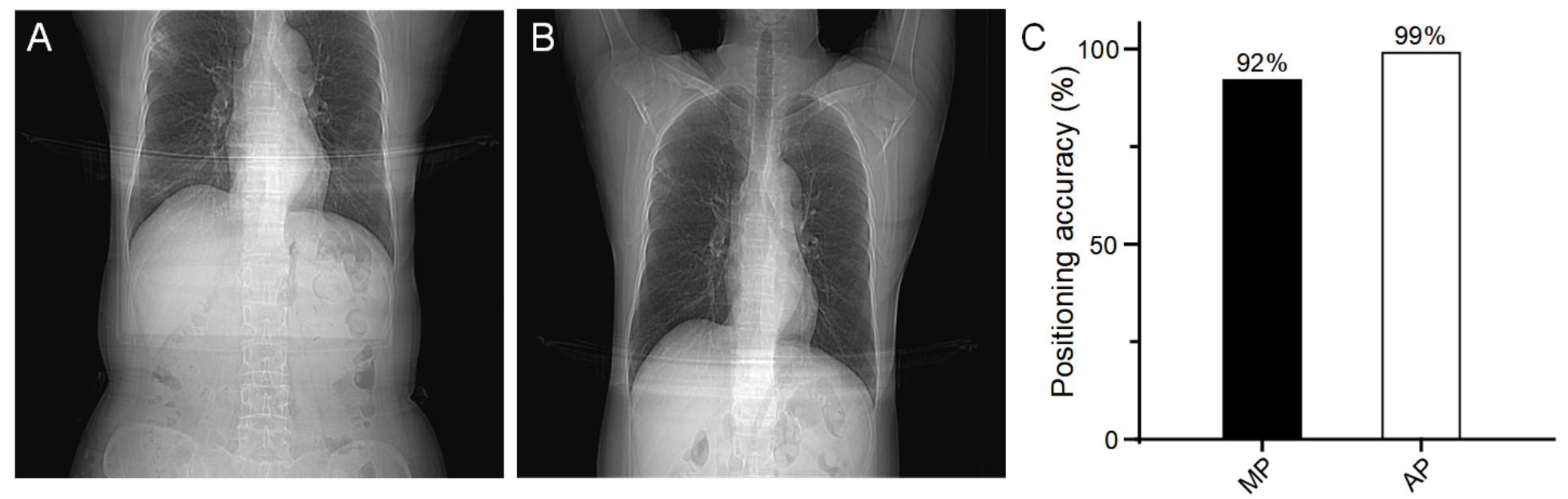

Figure 4

Comparison of positioning accuracy on chest CT topogram. (A) shows inaccurate chest topogram, in which lung field was incomplete displayed. (B) represents accurate chest CT topogram. (C) Comparison of positioning accuracy for chest topogram acquired by MP and AP. (Data were presented as $n(\%)$, where $n$ was the number of patients with complete CT scout image; $n=127$ each). 